Algebraic $\& \mathcal{G}$ Geometric Topology

Volume 2 (2002) 33-35

Published: 19 January 2002

ATG

\title{
A counterexample to a group completion conjecture of J C Moore
}

\section{ZBIGNIEW FiedorowiCZ}

\begin{abstract}
We provide a simple explicit counterexample to a group completion conjecture for simplicial monoids attributed to J C Moore.
\end{abstract}

AMS Classification 18G30; 55R35

Keywords Simplicial monoid, group completion

For a monoid $M$ let $U M$ denote the universal group generated by $M$, ie, the group generated by the set $\{[m] \mid m \in M\}$ modulo the relations $[m][n]=[m n]$. We shall refer to the natural homorphism $M \rightarrow U M$ as the group completion of $M$, having the universal property of being initial for homorphisms from $M$ into a group. If $M_{*}$ is a simplicial monoid, let $U M_{*}$ denote the simplicial group obtained by applying the functor $U$ degreewise. In his paper [4], D Puppe attributes the following conjecture to $\mathrm{JC}$ Moore and proves various special cases of it.

Conjecture If $M_{*}$ is a simplicial monoid such that $\pi_{0}\left(\left|M_{*}\right|\right)$ is a group, then group completion induces a homotopy equivalence

$$
\left|M_{*}\right| \longrightarrow\left|U M_{*}\right| \text {. }
$$

We will give a simple explicit counterexample to this conjecture below.

Lemma There is a discrete monoid $P$ whose classifying space $B P$ has the homotopy type of $S^{2}$.

Proof This follows immediately from a theorem of D MacDuff [3] (also proved in [2]), which shows that any connected CW homotopy type can be realized as the classifying space of a discrete monoid. However we will use the following explicit example: let $P$ be the 5 element monoid consisting of the unit 1 together with elements $\left\{x_{i j} \mid i, j=1,2\right\}$ which multiply according to the rule $x_{i j} x_{k \ell}=x_{i \ell}$. Since the elements $x_{i j}$ are idempotent,

$$
\pi_{1}(B P)=U P=1 .
$$


We then compute that $H_{*}(B P)=\operatorname{Tor}_{*}^{\mathbb{Z}[P]}(\mathbb{Z}, \mathbb{Z})=H_{*}\left(S^{2}\right)$ using the following projective resolution of right $\mathbb{Z}[P]$ modules

$$
0 \longrightarrow \mathbb{Z}\left[P_{1}\right] \oplus \mathbb{Z}\left[P_{2}\right] \longrightarrow \mathbb{Z}[P] \longrightarrow \mathbb{Z}\left[P_{1}\right] \longrightarrow \mathbb{Z} \longrightarrow 0,
$$

where $P_{i}=\left\{x_{i 1}, x_{i 2}\right\}$, the first map is given by inclusion, the second map is left multiplication by $x_{11}-x_{12}$, and the third map is the restriction of the augmentation.

Theorem There is a connected noncontractible simplicial monoid $M_{*}$ such that the group completion

$$
\left|M_{*}\right| \longrightarrow\left|U M_{*}\right|
$$

is null homotopic.

Proof Let $M_{k}$ denote the $k$-fold free product (ie, coproduct in the category of monoids) of the monoid $P$ with itself. Define the 0 -th and last face map to be the homorphism which kills the first, respectively last free summand, and for remaining $i$, let the $i$-th face be the $i$-th codiagonal. Define the $i$-th degeneracy to be the inclusion which misses the $i+1$-st free summand. It is easy to check that these specifications define a simplicial monoid $M_{*}$.

Let $B M_{*}$ be the simplicial topological space whose space of $k$-simplices is the classifying space $B M_{k}$. Then $B M_{*}$ has a simplicial subspace $S_{*}$, whose space of $k$-simplices is the $k$-fold wedge of $B P$ with itself. The first and last face drop the first and last wedge summand respectively, whereas the middle faces are given by fold maps. The degeneraces are given by inclusions of wedge summands. Since everything in degrees $>1$ is degenerate, the geometric realization of this simplicial space is the suspension $\Sigma B P \simeq \Sigma S^{2}=S^{3}$. As is shown in [2, Theorem 4.1], the inclusion $S_{*} \subset B M_{*}$ is a levelwise homotopy equivalence. Hence it follows that

$$
S^{3} \simeq\left|S_{*}\right| \simeq\left|B M_{*}\right|=B\left|M_{*}\right| .
$$

Since $M_{0}=1, \pi_{0}\left|M_{*}\right|=0$ is a group and so

$$
\left|M_{*}\right| \simeq \Omega B\left|M_{*}\right| \simeq \Omega S^{3} .
$$

(As noted in [4, page 382], this is an immediate consequence of [1].) Thus $M_{*}$ is noncontractible. On the other hand, $U M_{k}$ is the $k$-fold free product of $U P=1$ with itself, so $U M_{*}$ is the trivial simplicial group. 


\section{References}

[1] A Dold, Die geometrische Realisierung eines schiefen kartesichen Produktes, Archiv der Math. 9 (1958) 275-286

[2] Z Fiedorowicz, Classifying spaces of topological monoids and categories, Am. J. Math. 106 (1984) 301-350

[3] D MacDuff, On the classifying spaces of discrete monoids, Topology, 18 (1979) 313-320

[4] D Puppe, A theorem on semi-simplicial monoid complexes, Ann. of Math. 70 (1959) 379-394

Department of Mathematics, The Ohio State University

Columbus, $\mathrm{OH}$ 43210-1174, USA

Email: fiedorow@math.ohio-state.edu

URL: http://www.math.ohio-state.edu/ fiedorow/ 